\title{
Full Waveform Inversion with Multisource Frequency Selection of Marine Streamer Data
}

\author{
Yunsong Huang ${ }^{1,2}$ and Gerard T. Schuster ${ }^{1}$ \\ ${ }^{1}$ \{yunsong.huang, gerard.schuster\}@kaust.edu.sa \\ King Abdullah University of Science and Technology, \\ 4700 King Abdullah University of Science and Technology \\ Thuwal, Kingdom of Saudi Arabia 23955-6900 \\ 2 yunsongh@lanl.gov \\ Los Alamos National Laboratory, \\ EES-17, mail stop D446 \\ Los Alamos, NM 87545
}

(September 30, 2017)

Running head: FWI Multisource Frequency Selection

\begin{abstract}
The theory and practice of multisource full waveform inversion of marine supergathers are described with a frequency-selection strategy. The key enabling property of frequency selection is that it eliminates the crosstalk among sources, thus overcoming the aperture mismatch of marine multisource inversion. Tests on multisource full waveform inversion of synthetic marine data and Gulf of Mexico data show speedups of $4 \times$ and $8 \times$, respectively, compared to conventional full waveform inversion.
\end{abstract}

This article has been accepted for publication and undergone full peer review but has not been through the copyediting, typesetting, pagination and proofreading process, which may lead to differences between this version and the Version of Record. Please cite this article as doi: $10.1111 /$ gpr.12588

This article is protected by copyright. All rights reserved. 


\section{INTRODUCTION}

Full-waveform inversion (FWI) inverts seismic traces for Earth's rock properties, and has the potential to provide velocity models with high resolution. Excellent exposition on this topic can be found in Virieux et al. (2017) and Fichtner (2011). A major challenge with FWI is the high computational cost associated with the multiple iterations of forward and backward propagation of the wavefields. The number of such modeling operations, involving a large amount of seismic data, is proportional to not only the number of iterations, but also the number of seismic shots.

A partial remedy to the computational cost of the wavefield modeling is to reduce the number of effective seismic shots by forming 'supergathers'. A supergather is formed by encoding and summing up shot gathers from multiple seismic sources. This is referred

to as the multisource approach (Morton and Ober, 1998; Jing et al., 2000; Zhang et al., 2003; Etgen, 2005; Vigh and Starr, 2008; Ben-Hadj-Ali et al., 2009; Krebs et al., 2009; Tang and Biondi, 2009; Godwin and Sava, 2010; Boonyasiriwat and Schuster, 2010; Huang and Schuster, 2012; Dai et al., 2013). For example, 1000 shot gathers can be divided into 50 supergathers, each containing 20 shot gathers. The multisource approach enhances the computational efficiency ostensibly by about $20 \times$ in this example, but the penalties are crosstalk noise (Romero et al., 2000) in the FWI gradient and the resultant inaccuracy in the inverted velocity model. To mitigate this crosstalk noise, the encoding scheme is distinct at each iteration of an iterative inversion method, such as FWI or least-squares migration (LSM), so that as the iterations proceed the crosstalk noise between different shots in a supergather dwindles (Dai et al., 2012). Continuing the previous example, if compared to a standard iterative method, the multisource approach requires $4 \times$ the number of iterations 
in order to reduce the noise to a comparable level, the gain in computational efficiency is about $20 / 4=5 \times$.

A succinct mathematical overview of encoded FWI is now presented. The formulas associated with the FWI misfit gradient $\nabla_{\mathbf{v}} \epsilon$ are given below:

$$
\begin{aligned}
\mathbf{d}^{\text {pred }} & =f(\mathbf{v}) \simeq \overbrace{f\left(\mathbf{v}^{(k)}\right)}^{\mathbf{f}^{(k)}}+\mathbf{L m}, \\
\text { misfit } \epsilon & =\frac{1}{2}\left\|\mathbf{d}^{\text {pred }}-\mathbf{d}^{\text {obs }}\right\|^{2}, \\
& \simeq \frac{1}{2}\left\|\mathbf{f}^{(k)}+\mathbf{L m}-\mathbf{d}^{\text {obs }}\right\|^{2}, \\
\left.\nabla_{\mathbf{v}} \epsilon\right|_{\mathbf{m} \rightarrow 0} & =\mathbf{L}^{\dagger}\left[\mathbf{f}^{(k)}-\mathbf{d}^{\text {obs }}\right] .
\end{aligned}
$$

Here, $\mathbf{d}^{\text {pred }}$ and $\mathbf{d}^{\text {obs }}$ represent the predicted and the observed seismic data; $f(\mathbf{v})$ represents the wavefield forward-modeling, for a supergather, dependent nonlinearly on the velocity model $\mathbf{v}$; and $\mathbf{m}$ represents an infinitesimal model perturbation. Linearizing $f\left(\mathbf{v}=\mathbf{v}^{(k)}+\mathbf{m}\right)$ with respect to $\mathbf{m}$ leads to the right hand of equation 1 , where $\mathbf{L}$ represents the Fréchet derivative. (The dependence of $\mathbf{L}$ on $\mathbf{v}$ is suppressed for succinctness, whereas in LSM such dependence is immaterial because $\mathbf{v}$ does not change over iterations.) The superscripts ${ }^{(k)}$ and ${ }^{\dagger}$ denotes association with the $k^{\text {th }}$ iteration and adjoint, respectively.

The encoding and blending for a supergather in the frequency domain can now be expressed as

$$
\mathbf{L}=\sum_{i} P_{i} \mathbf{L}_{i}, \quad \mathbf{d}^{\mathrm{obs}}=\sum_{i} P_{i} \mathbf{d}_{i}^{\mathrm{obs}}, \quad \mathbf{f}^{(k)}=\sum_{i} P_{i} \mathbf{f}_{i}^{(k)},
$$

where $\mathbf{L}_{i}, \mathbf{d}_{i}^{\text {obs }}$, and $\mathbf{f}_{i}^{(k)}$ represent, respectively, the Fréchet derivative for the $i^{\text {th }}$ shot gather, the $i^{\text {th }}$ observed common shot gather, and the forward modeled wavefield for the $i^{\text {th }}$ shot in the supergather, and $P_{i}$ is the encoding operator. The role of $P_{i}$ is to produce dissimilarity 
between shot gathers so that they are mutually uncorrelated in some sense. For example, if $P_{i}$ 's are mutually independent 0 -mean random variables (RV) taking values \pm 1 , then ideally $\left\langle\mathbf{L}_{i}^{\dagger} P_{i}^{\dagger} P_{j} \mathbf{L}_{j}\right\rangle=\mathbf{L}_{i}^{\dagger} \mathbf{L}_{j} \delta_{i j}$, where $\delta_{i j}$ is the Kronecker delta and $\langle\ldots\rangle$ denotes statistical expectation over the RV $P_{i}{ }^{\prime}$ s (Schuster et al., 2011).

The velocity-model update associated with the encoded supergather is given by

$$
\begin{aligned}
\mathbf{v}^{(k+1)}-\mathbf{v}^{(k)} & =-\alpha^{(k)} \nabla_{\mathbf{v}} \epsilon=\alpha^{(k)} \mathbf{L}^{\dagger}\left[\mathbf{d}^{\text {obs }}-\mathbf{f}^{(k)}\right], \\
& =\alpha^{(k)} \sum_{i} \mathbf{L}_{i}^{\dagger} P_{i}^{\dagger} P_{i}\left[\mathbf{d}_{i}^{\text {obs }}-\mathbf{f}_{i}^{(k)}\right]+\alpha^{(k)} \sum_{i} \sum_{j \neq i} \mathbf{L}_{i}^{\dagger} P_{i}^{\dagger} P_{j}\left[\mathbf{d}_{j}^{\text {obs }}-\mathbf{f}_{j}^{(k)}\right], \\
& =\sum_{i} \overbrace{\alpha^{(k)} \mathbf{L}_{i}^{\dagger}\left[\mathbf{d}_{i}^{\text {obs }}-\mathbf{f}_{i}^{(k)}\right]}^{\text {update due to } i^{\text {th }} \text { shot }}+\text { crosstalk, }
\end{aligned}
$$

where $\alpha^{(k)}$ represents the step size at the $k^{\text {th }}$ iteration. As mentioned, the encoding functions change at each iteration, and for a sufficient number of iterations the crosstalk terms incoherently sum together to become small compared to the coherently stacked velocity updates (Dai et al., 2012).

There are a number of different encoding strategies.

1. Random Time Series: $P_{i}$ represents a white-noise series which, in the time domain, is convolved with each trace of the $i^{\text {th }}$ shot gather (Romero et al., 2000). The resulting shot gathers are then blended together to get one supergather, and this supergather is migrated by a wave-equation migration method for many sources. This procedure can be repeated at each iteration with a different set of white-noise sources, and the migration images are stacked together.

2. Random Polarity: $P_{i}$ is a random scalar variable with value either +1 or -1 and 
multiplies each trace in the $i^{\text {th }}$ shot gather (Krebs et al., 2009). These randomly polarized shot gathers are then blended together to get one supergather.

3. Random Time Shift: Each trace in the $i^{\text {th }}$ shot gather is convolved with $\delta\left(t-\tau_{i}\right)$ such that $\tau_{i}$ is a random time shift (Dai and Schuster, 2009; Tang and Biondi, 2009). These shifted traces are then blended together to get one supergather.

4. Plane Wave Encoding: Shot gathers are time shifted linearly with respect to sourcereceiver offset and summed together to form a plane-wave source with a specified slowness value (Zhang et al., 2003; Etgen, 2005; Vigh and Starr, 2008).

With the help of data interpolation and the reciprocity property, plane-wave encoding can be made applicable for marine acquisition geometries (Dai and Schuster, 2013). Except in this instance, the above encoding strategies are all efficiently suited for land data where the receiver spread is fixed for each shot, but not for marine data with a receiver array that moves with each shot.

As an illustration, Figure 1(a1) shows two shot gathers to be blended, where one shot is at the red source and the other is at the dark-blue source; blending these two gathers together, e.g., adding together the red and blue traces recorded at the same geophone, gives the 2-shot supergather denoted as $\mathbf{d}^{\text {obs }}$. Typical of marine surveys, the receiver array is at a different offset for either source so that only certain receivers are selectively listening for the red shot but not for the dark blue shot at the unshared receiver positions. In stark contrast, a finite-difference simulation of two simultaneous sources (a red source and a dark blue source) will compute traces everywhere on the surface that are a superposition of the wavefields from both sources. Namely, the predicted 2-shot supergather $\mathbf{d}^{\text {pred }}$ generated by a finite-difference solution of the wave equation does not discriminate and generates 
traces at every receiver, as shown in Figure 1(a2). Hence, there will be discrepancies between the predicted and observed traces at the unshared receiver positions (indicated by the dashed ovals in Figure 1). We denote this problem in multisource FWI as the aperture mismatch problem, where the observed supergather is for a blended marine survey while the predicted supergather is for a blended land survey.

The aperture mismatch will lead to a non-zero misfit function (equation 2), even if the exact velocity model is used for prediction. The remedy to this mismatch is to use an encoding function in the multisource finite-difference modeling that only activates specified receivers for any one shot. This orthogonal encoding strategy, referred to interchangeably as 'frequency-division' or 'frequency-selection', was developed by Huang and Schuster (2012) for wave equation migration. The key idea of this orthogonal encoding strategy is to allow $P_{i}$ to be a narrow bandpass filter which, in the time domain, is convolved with each trace of the $i^{\text {th }}$ shot gather. The passbands do not overlap, so the time-domain representations of $P_{i}$ and $P_{j}$ convolved with one another are zero unless $i=j$. These filtered shot gathers are then blended together to get one supergather. Now this encoding strategy will be tested for FWI.

The first part of the paper provides the theory for multisource FWI with frequency selection, and is followed by results from tests on synthetic and field data. Speedups ranging from $4 \times$ to $8 \times$ compared to conventional FWI are obtained. The last part presents a summary. 


\section{THEORY}

The formulas for multisource FWI are given in equations 4, 6, and 7. The frequencyselection encoding scheme is described in Huang and Schuster (2012), and is summarized by the following steps.

1. Figure 1(a) illustrates the problem, and the first step is to assign a non-overlapping frequency spectrum to each of the sources. In Figure 1(b), the cyan (red) source is bandlimited around $20 \mathrm{~Hz}(10 \mathrm{~Hz})$ during the multisource simulation and the supergather is computed.

2. In Figure 1(c), a bandpass filter is applied to decode the (b) supergather so that the cyan traces can be separated from the red traces. In each shot gather, only traces recorded by receivers in a hypothetical marine survey are retained while the others are muted.

3. In Figure 1(d), the decoded and muted traces are blended together to give the bandlimited marine supergather. This procedure is iterated in equation 6, except that a unique non-overlapping frequency is iteratively assigned to each source. For a sufficient number of iterations, the full bandwidth of the data is employed at each source.

[Figure 1 about here.]

To apply the frequency-selection to a finite-difference time-domain (FDTD) simulation, causal sine waves are injected at the source positions (and at receivers when backpropagating the data misfit). This causality shows up as transients in recorded sinusoidal 
seismograms, causing spectral leakage. To mitigate this problem, assuming the impulse response of the Earth is active only within $n t$ time samples, we run the FDTD simulations over $2 n t$ time samples, and keep the responses only within the last $n t$ time samples, as illustrated in Figure 2. The responses in the last $n t$ time samples are nearly identical to what is obtained by running the simulation over $n t$ time samples with an input of infinitely long sine waves of period $n t$ time samples. Such sine waves are the basis functions of the discrete-time Fourier transform (DTFT), if performed on the data obtained from the FDTD simulation over $n t$ time samples using a time-limited source wavelet.

[Figure 2 about here.]

[Figure 3 about here.]

This transient-reduction scheme also applies when back-propagating the residual wavefield in forming the gradient of FWI, as illustrated in Figure 3. Note that only the times slices between time-steps $n t+1$ and $2 n t$ of the source wavefield need to be reconstructed from the FDTD boundary. The total number of time steps of FDTD propagation thus equals $2 n t+n t+2 n t=5 n t$, where the two $2 n t^{\prime}$ s denote the numbers of time steps for the forward-propagation and back-propagation of the source and residual wavefields, respectively, while the $n t$ is for reconstruction of the latter half of the source wavefield. This compares to $3 n t$ time steps in the standard approach in computing the gradient.

In numerical optimization, after the gradient is computed, Brent's method (Press et al., 2007) is used for the line search. This takes on average 5 function evaluations, namely, 5 passes of forward-propagation of the source wavefield. Therefore in the standard approach, $3 n t+5 \cdot n t=8 n t$ time steps of FDTD propagation are required per iteration of FWI, whereas 
in our transient-reduction scheme, $5 n t+5 \cdot 2 n t=15 n t$ time steps of FDTD propagation are necessary. Roughly, per iteration the latter costs about twice as much as the former does (Dai et al., 2013).

\section{NUMERICAL RESULTS}

\section{Synthetic Data}

The full waveform inversion multiscale frequency-selection (FWIMFS) method is tested on synthetic data computed for the SEG/EAGE salt model with a marine geometry. The model is decimated by a factor of $3 \times 3$ for less overall computational time, as shown in Figure 4(b). The source wavelet is a Ricker wavelet peaking at $8 \mathrm{~Hz}$. There are 60 shot gathers evenly distributed across the top of the model with the shot spacing of $82.3 \mathrm{~m}$, the receiver spacing is $27.4 \mathrm{~m}$, and the line length is $2.3 \mathrm{~km}$. In FWIMFS, all the 60 shot gathers are included in one supergather.

The FWI method uses a preconditioned conjugate gradient method, where the acoustic forward and backward solvers are a finite-difference solution to the 2D space-time wave equation of constant density. The FDTD algorithm is second-order accurate in time and fourth-order accurate in space, denoted as $O(2,4)$. The source wavelet for the proposed frequency-selection method is a pure cosine wave, also employed in Nihei and Li (2007) and Sirgue et al. (2008), at a selected frequency.

\section{[Figure 4 about here.]}

The starting model is shown in Figure 4(a) and the standard FWI tomogram after 69 iterations is shown in Figure 4(d). The FWIMFS strategy produces the tomogram shown 
in Figure 4(c). This result required 439 iterations to achieve the same accuracy as the Figure 4(d) result in 69 iterations. For this test, in terms of the convergence of waveformand velocity-error, to show that FWIMFS with 439 iterations roughly equates the standard FWI with 69 iterations, we seek to equate each pair of convergence curves (Figure 5) by shrinking the horizontal axes for the red dashed curves (i.e., for FWIMFS) by $439 / 69=6.362$ times. (The convergence curves are rendered this way because our vision is better at judging equality than quantifying a specific ratio.)

[Figure 5 about here.]

The speedup $\rho$ of FWIMFS over standard FWI can be estimated as $\rho=\frac{t_{\text {standard FWI }}}{t_{\mathrm{FWIMFS}}}=$ $\frac{69 \times 60}{439 \times 2}=4.72$, where the ' $\times 2$ ' in the denominator is explained in the paragraph just before this section, and the ' $\times 60$ ' in the numerator is because the computational time for the standard FWI scales with the number of shot gathers. To be conservative, we arrive at the the speedup of $4 \times$. This compares to the $8 \times$ speedup reported by Huang and Schuster (2012) for reverse-time migration (RTM). The reason for this discrepancy is that the implementation in the time domain suffers from a $2 \times$ overhead in order to reduce the effect of transient noise in the simulated sinusoidal seismograms.

\section{Gulf of Mexico Streamer Dataset}

The FWIMFS is then tested on a Gulf of Mexico (GOM) streamer dataset. We include 496 shots with a shot interval of $37.5 \mathrm{~m}$. The source-receiver offset ranges from $198 \mathrm{~m}$ to $6 \mathrm{~km}$, with a receiver spacing of $12.5 \mathrm{~m}$. The trace length is $7 \mathrm{~s}$, with a sampling interval of $2 \mathrm{~ms}$.

This test consists of the following steps: 
1. Estimate the source wavelet, by integrating the direct wave.

2. Use a geometrical spreading correction to convert the $3 \mathrm{D}$ physical data to $2 \mathrm{D}$, on which grid the simulations are run.

3. Obtain an initial-velocity model, shown in Figure 9(a), by combining traveltime tomography and the velocity from semblance analysis, referred to as 'traveltime tomography'.

4. Implement a multiscale strategy (Bunks et al., 1995) with FWIMFS.

5. To validate the accuracy of the tomogram, compute the RTM image and common image gather using the tomogram velocity.

[Figure 6 about here.]

[Figure 7 about here.]

The source wavelet is estimated as follows. 1) Stack the direct waves from a common offset gather; 2) integrate the stacked trace along time; and 3) set its direct current (DC) to 0 . The reason for integration is because the received direct wave is proportional to the time derivative of the source wavelet, as illustrated in Figure 6. The estimated source wavelet is plotted in Figure 7(a); a bandpass filtered version (filtered with a Ricker wavelet of peak frequency $10 \mathrm{~Hz}$ ) is plotted in Figure 7(b). There are 210 frequency components supporting the power spectrum curve plotted in Figure 7(c). As the power is very small at the tails, rather than assigning each frequency component individually, we group them into 62 frequency intervals, denoted by alternating colors in Figure 7(c). The intervals are wider near the tails, so that the area under the curve in each interval is approximately equalized. 
Each interval of frequencies is selected as one entity in this frequency-selection scheme. The $3 \mathrm{D}$ to $2 \mathrm{D}$ conversion is achieved by multiplying the data spectra by $\sqrt{i / \omega}$ (Barton, 1989) and then gaining in the time domain by $\sqrt{t}$.

Aspects of frequency-selection and stochastic optimization

[Figure 8 about here.]

In step 4, for the multisource frequency-selection (MFS), a natural encoding strategy to consider is Quasi-Monte Carlo encoding(Boonyasiriwat and Schuster, 2010). We present such a strategy motivated as follows. It is desirable that every source has a chance of evenly sampling the frequency components assigned to it over the many iterations of FWIMFS. An example of 10 frequency components and 4 iteration steps for a particular source might select the indices like 9,2,5,7, where each number denotes a frequency index; 4 iterations give 4 numbers. An undesirable example is 3,2,2,1, which over-represents the low frequency index 2 , omitting the medium and high-frequency components. Moreover, nearby sources tend to illuminate an overlapping region of subsurfaces. Therefore if their frequency content differs, then they as a whole would cover a wider range of frequency components. For instance, the neighbors emitting sine waves of frequency with indices like $2,9,5,7$ would be less desirable than those emitting a wider band of frequencies with indices $1,10,3,8$.

To achieve this end, we introduce repellent Coulomb forces between 2D point charges, each point $\left(\omega_{j}, s_{i}\right)$ denoting a mapping from $\omega_{j}$ to source $s_{i}$. This electrostatic system then settles, simulated through greedy optimization, into a low-energy configuration, in which all charged points spread out as much as possible. Examples of this encoding strategy are 
shown in the left column of Figure 8, which appear more uniformly distributed than the counterparts of the standard random permutation shown in the right column.

As the specific frequency-selection code changes over iterations of FWI, this falls in the realm of stochastic optimization (Spall, 2003). While the convergence of a line search in stochastic optimization is still a research problem, we adopt a hybrid approach. Run a gradient descent method with line search for the first $K_{0}$ iteration steps of FWI, then switch to a stochastic gradient descent method, where the step size $\propto 1 / k, k$ being the iteration step index. This is a robust algorithm that converges almost surely to a local minimum (Kiwiel, 2001). Moreover, since no line search is necessary, this algorithm per step is more economic than the alternatives that require accurate line search. The problem remains in choosing the appropriate constant coefficient for this step size formula. The recipe is, first, identify the smallest $\eta, \eta_{\min }$, resulting from the first $K_{0}$ steps of line search, expressed in the form

$$
\|\Delta \mathbf{x}\|=\eta\|\nabla J(\mathbf{x})\|
$$

Here, $\mathbf{x}$ is the unknown parameter vector, such as the velocity model; $\Delta \mathbf{x}$ is the update of $\mathbf{x}$ at a step; and $\nabla J$ represents the gradient of the objective function. Then, the constant coefficient of the step size formula can be fixed accordingly, as

$$
\Delta \mathbf{x}^{(k)}=-\eta_{\min } \frac{K_{0}}{k} \nabla J\left(\mathbf{x}^{(k)}\right), \quad \text { for } k=K_{0}+1, \ldots
$$

A similar recipe for determining the step size of stochastic gradient descent is suggested in Bottou and Bousquet (2011).

As mentioned at the end of THEORY, 5 function evaluations are required on average by 
Brent's method for line search. In contrast, none is required in a stochastic gradient descent method. Consequently, per iteration step of the latter method, the computational cost is only due to the gradient computation, which requires, with the transient-reduction scheme, $5 n t$ FDTD propagation steps. This compares to $8 n t$ in the standard approach, which, taking advantage of the conjugate gradient (CG) method, needs an accurate line search, and therefore explains the associated overhead. This computationally inexpensive stochastic gradient descent approach translates to more iterations and therefore more frequencyselection codes in use.

To further reduce the amount of stochasticity in the gradient, we empirically adopt averaging of two successive gradient calculations. Namely, perform the multisource encoding and gradient computation twice, then stack the gradients. This will double the computational cost. So a tradeoff exists between the size of the number of gradients in the average and the convergence speed of the stochastic gradient descent. This averaging (also known as the mini-batch) scheme applies to all 8 supergathers, which are formed by dividing up the 496 shot gathers.

We start the inversion with the data bandpass filtered from $0-6 \mathrm{~Hz}$. The initial velocity model is decimated to a discrete grid size of $n z \times n x=51 \times 376$. At later iterations the band is widened to $15 \mathrm{~Hz}$, and the model is upsampled (with interpolation) to $n z \times n x=101 \times 752$. Finally the frequency band covers 0 to $25 \mathrm{~Hz}$, and the velocity model is of grid size $n z \times n x=201 \times 1504$. We only use standard FWI for the first two cases, because the amount of computation is negligible compared to the third case. For example, the second case has a quarter of the model size, half of the time samples (because of doubled time sampling interval), and half the number of shots (due to downsampling). Therefore the computational load per iteration of the second case is only $1 / 16$ of the third. 
Results of the GOM dataset

As shown in Figures 9(b) and (c), the tomograms computed by standard FWI after 20 iterations and by FWIMFS after 71 iterations appear very similar. The associated computational cost of the latter case is $1 / 8$ of that of the former. In both cases, there appear to be some regions of low-velocity anomalies. One is a horizontal layer at a depth of $0.75 \mathrm{~km}$, where the velocity is slower than in water. This could be due to out-of-plane wavepaths modeled in 2D, resulting in underestimating the velocity, or it indicates unconsolidated sediments. Other low-velocity anomalies seem to lie along the wavepaths of diving waves. This arises due to cycle-skipping of some of the diving waves. Such velocities can be elevated by manual intervention, a trial move to be accepted if it overcomes some cycle-skipping. Rather than going into details of improving the FWI results, this paper aims at demonstrating that FWIMFS produces essentially the same results as standard FWI does, with the benefit of a reduction in the computational cost. How we massage the velocity model to address the cycle-skipping is a separate research project.

[Figure 9 about here.]

[Figure 10 about here.]

In order to see how the quasi-Monte Carlo encoding scheme accelerates the inversion, Figure 10 shows the velocity differences between the tomograms obtained using the two alternative encoding schemes: standard random permutation versus quasi-Monte Carlo. Surprisingly, the two velocity models are almost identical. The other results, such as RTM images and common-image gathers (CIGs), appear indistinguishable, and therefore further comparisons between the two are omitted. This implies that the FWIMFS is very robust with 
regard to specific random codes of frequency-selection. Figure 11 plots the convergence curves of the various approaches. The reason why the red curve lies slightly above the green one is explained as follows. By quasi-Monte Carlo encoding, the new multisource data presented to the inversion algorithm is very different from what it has seen in the past, because this data contains entirely new frequency components. In contrast, by standard random permutation, some frequency components may have been used earlier, and thus the new multisource data is not entirely new. So the algorithm using standard random permutation can fare better at fitting the data.

[Figure 11 about here.]

[Figure 12 about here.]

To probe the qualities of FWI, RTM images are shown in Figure 12, with zoomed views shown in Figure 13. Note that the results of standard FWI and of FWIMFS appear identical. In the three zoomed views, the FWI results seem more focused, showing better continuity of reflectors. In the center of the RTM images, around $(X$, Depth $)=(9.5,1.5) \mathrm{km}$, however, the traveltime tomogram offers better focusing than FWI tomograms. This is perhaps due to the overhanging low-velocity anomalies in the FWI tomograms, as remarked on Figure 9.

[Figure 13 about here.]

[Figure 14 about here.]

Comparing the CIGs in Figure 14, we see flatter events on the right part, delimited by the red box. This coincides with the observations about the RTM images in that the FWI results fare better (in terms of more focused and continual reflectors) on the right part of 
the RTM image. In the green box of the CIGs, although the FWI results are flatter than the traveltime counterpart, the FWI velocity is over-corrected to be too small. This is indicated by the events curving up with larger source-midpoint offset (larger offset is on the left side of each CIG strip). This supports the idea that the overhanging low-velocity anomalies are not authentic.

[Figure 15 about here.]

Figures 15 and 16 show CSGs from two sources, obtained by various methods. The match between the FWI results and the observed ones is generally good, although cycle skipping can be found at offsets $=3.8 \mathrm{~km}$ and $6 \mathrm{~km}$, while comparing panels (a) and (c) in Figure 15 .

[Figure 16 about here.]

\section{CONCLUSIONS}

Multisource full waveform inversion of supergathers for marine data is implemented with a frequency-selection strategy. The key enabling property of frequency selection is that it eliminates the crosstalk among sources, thus overcoming the aperture mismatch of marine multisource inversion. This method is now extended to the finite-difference time-domain from the previous implementation with phase shift migration. Tests on multisource full waveform inversion of synthetic marine data and Gulf of Mexico data show speedups of $4 \times$ and $8 \times$, respectively, compared to conventional full waveform inversion. 


\section{ACKNOWLEDGMENTS}

We wish to thank the sponsors of the Center for Subsurface Imaging and Fluid Modeling (CSIM) at KAUST for their support. 


\section{REFERENCES}

Barton, G., 1989, Elements of Green's functions and propagation: potentials, diffusion, and waves: Oxford University Press.

Ben-Hadj-Ali, H., S. Operto, and J. Virieux, 2009, Three-dimensional frequency-domain full waveform inversion with phase encoding: SEG Technical Program Expanded Abstracts, $2288-2292$.

Boonyasiriwat, C., and G. Schuster, 2010,3D multisource full-waveform inversion using dynamic quasi-monte carlo phase encoding: SEG Technical Program Expanded Abstracts, 1044-1049.

Bottou, L., and O. Bousquet, 2011, The tradeoffs of large-scale learning: Optimization for Machine Learning, 351.

Bunks, C., F. M. Saleck, S. Zaleski, and G. Chavent, 1995, Multiscale seismic waveform inversion: Geophysics, 60, 1457-1473.

Dai, W., P. Fowler, and G. T. Schuster, 2012, Multi-source least-squares reverse time migration: Geophysical Prospecting, 60, 681-695.

Dai, W., Y. Huang, and G. T. Schuster, 2013, Least-squares reverse time migration of marine data with frequency-selection encoding: Geophysics, 78, S233-S242.

Dai, W., and G. T. Schuster, 2009, Least-squares migration of simultaneous sources data with a deblurring filter: SEG Technical Program Expanded Abstracts, 2990-2994.

—_, 2013, Plane-wave least-squares reverse-time migration: GEOPHYSICS, 78, S165S177.

Etgen, J. T., 2005, How many angles do we really need for delayedshot migration?: SEG Technical Program Expanded Abstracts, 1985-1988.

Fichtner, A., 2011, Full seismic waveform modelling and inversion: Springer. 
Godwin, J., and P. Sava, 2010, Blended source imaging by amplitude encoding: SEG Technical Program Expanded Abstracts, 3125-3129.

Huang, Y., and G. Schuster, 2012, Multisource least-squares migration of marine streamer and land data with frequency-division encoding: Geophysical Prospecting, 60, 663-680.

Jing, X., C. J. Finn, T. A. Dickens, and D. E. Willen, 2000, Encoding multiple shot gathers in prestack migration: SEG Technical Program Expanded Abstracts, 786-789.

Kiwiel, K. C., 2001, Convergence and efficiency of subgradient methods for quasiconvex minimization: Mathematical Programming, 90, 1-25.

Krebs, J. R., J. E. Anderson, D. Hinkley, R. Neelamani, S. Lee, A. Baumstein, and M.-D. Lacasse, 2009, Fast full-wavefield seismic inversion using encoded sources: Geophysics, 74, WCC177-WCC188.

Morton, S. A., and C. C. Ober, 1998, Faster shot-record migrations using phase encoding: SEG Technical Program Expanded Abstracts, 1131-1134.

Nihei, K., and X. Li, 2007, Frequency response modelling of seismic waves using finite difference time domain with phase sensitive detection (td-psd): Geophysical Journal International, 169, 1069-1078.

Press, W. H., S. A. Teukolsky, W. T. Vetterling, and B. P. Flannery, 2007, Numerical recipes 3rd edition: The art of scientific computing, 3 ed.: Cambridge University Press.

Romero, L. A., D. C. Ghiglia, C. C. Ober, and S. A. Morton, 2000, Phase encoding of shot records in prestack migration: Geophysics, 65, 426-436.

Schuster, G. T., X. Wang, Y. Huang, W. Dai, and C. Boonyasiriwat, 2011, Theory of multisource crosstalk reduction by phase-encoded statics: Geophysical Journal International, 184, 1289-1303.

Sirgue, L., J. Etgen, and U. Albertin, 2008, 3d frequency domain waveform inversion using 
time domain finite difference methods: Presented at the Proceedings of the 70th EAGE Conference and Exhibition, Rome, Italy.

Spall, J., 2003, Introduction to stochastic search and optimization: estimation, simulation, and control: John Wiley and Sons.

Tang, Y., and B. Biondi, 2009, Least-squares migration/inversion of blended data: SEG Technical Program Expanded Abstracts, 2859-2863.

Vigh, D., and E. W. Starr, 2008, 3d prestack plane-wave, full-waveform inversion: Geophysics, 73, VE135-VE144.

Virieux, J., A. Asnaashari, R. Brossier, L. Mtivier, A. Ribodetti, and W. Zhou, 2017, An introduction to full waveform inversion, in Encyclopedia of Exploration Geophysics: Society of Exploration Geophysicists, R1-1-R1-40.

Zhang, Y., J. Sun, C. Notfors, S. Gray, L. Chernis, and J. Young, 2003, Delayed-shot 3D prestack depth migration: SEG Technical Program Expanded Abstracts, 1027-1030. 


\section{LIST OF FIGURES}

1 The solution to the acquisition misfit problem. . . . . . . . . . . . 23

2 Doubling the simulation time of FDTD to mitigate the transient. . . . . . . . 24

3 Transient-reduction in forming the gradient of FWI. . . . . . . . . . . . 25

4 FWI of alternative methods on the SEG/EAGE salt model. . . . . . . . . . . 26

5 Normalized waveform and velocity errors over iterations. . . . . . . . . . 27

6 The direct wave and free-surface reflection combine together to give a detected signal. . . . . . . . . . . . . . . . . . 28

7 The estimated source wavelet and spectral division. . . . . . . . . . . . . . 29

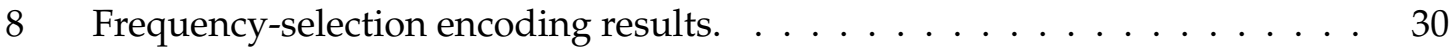

9 FWI results for the Gulf of Mexico (GOM) dataset. . . . . . . . . . . . . . . . 31

10 Difference of FWI velocity models obtained using the two frequency-selection encoding means. . . . . . . . . . . . . . . . . 32

11 Reduction of data misfit plotted against FWI iterations. . . . . . . . . . . . 33

12 RTM images of the tomograms. . . . . . . . . . . . . . . 34

13 Zoomed views of the RTM images. . . . . . . . . . . . . . . . 35

14 Shot domain CIG . . . . . . . . . . . . . . . . . . . . . 36

15 Common shot gather (CSG) of various approaches for GOM data of one source at $X=6 \mathrm{~km} \ldots \ldots \ldots \ldots \ldots \ldots$

16 CSG of various approaches for GOM data of one source at $X=12 \mathrm{~km}$. . . . 38 


\section{Aperture Mismatch Problem with Multisource Marine Data and a Solution with Frequency-Selection Encoding}

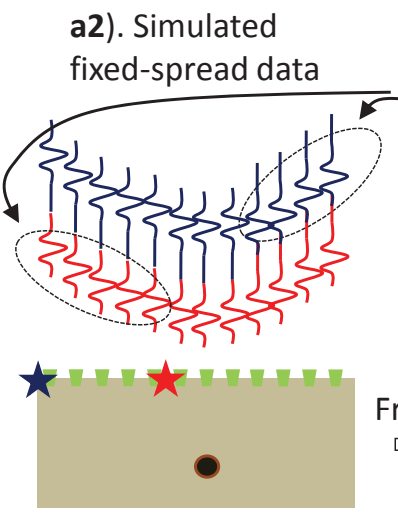

b). Simulation with

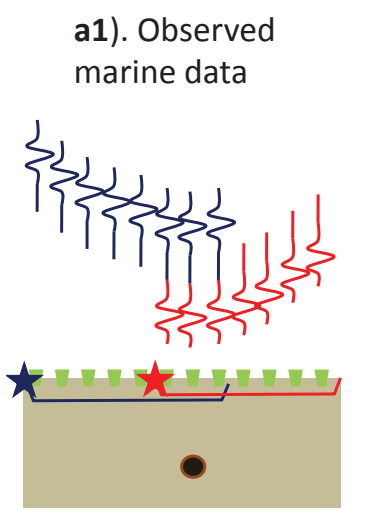

a1). Observed

marine data

d). Blend $\rightarrow$ simulated marine data

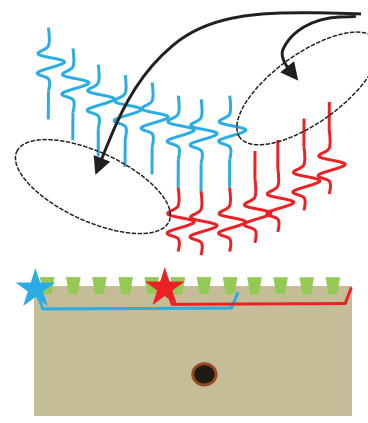

wrong misfit

eliminated

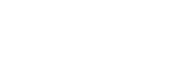

Figure 1: The solution to the acquisition misfit problem illustrated in panels (a1) and (a2), is given by the following steps: (b). the cyan (red) source is bandlimited around $20 \mathrm{~Hz}$ $(10 \mathrm{~Hz})$ during the multisource simulation; (c). a bandpass filter is applied to decode these traces so that the cyan traces can be separated from the red ones. Muting of the specified traces for a marine geometry is applied. (d). The decoded and muted traces are blended together to give the bandlimited marine supergather. Now, there is no aperture mismatch between the simulated and observed supergathers, except that the frequency channels of the former are a subset of those of the latter. 


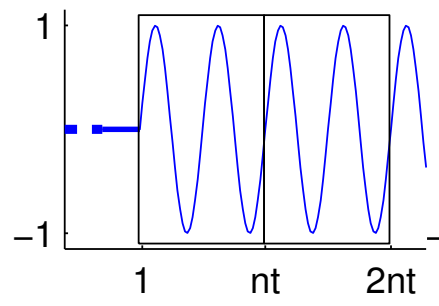

(a)

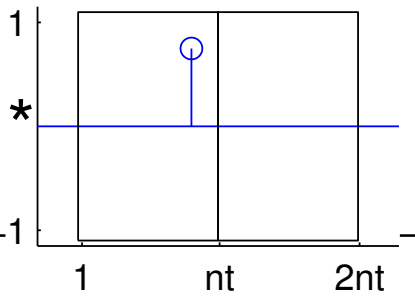

(b)

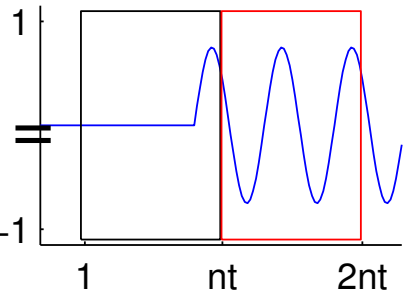

(c)

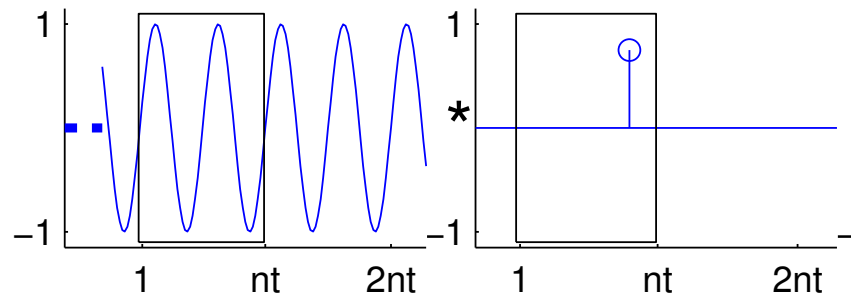

(e)

(f)

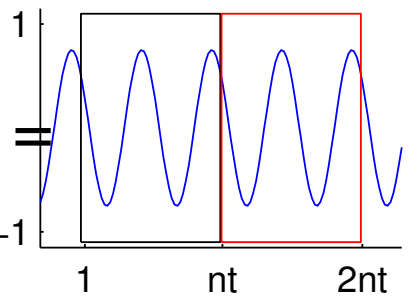

(g)

Figure 2: Doubling the simulation time of FDTD to mitigate the transients when causal sine waves are injected at the inputs, e.g., sources or receivers. Discrete time is assumed here, i.e., the time interval $\Delta t$ is silent. If a (a) causal sine wave $u(t) \sin (2 q \pi t / n t)$, where $u(t)$ represents a unit-step function, is convolved with (b) a delayed impulse, the output is a delayed version of the input signal, plotted in (c), where the black box contains the transient, while the red box contains the steady-state. If $\sin (2 q \pi t / n t)$, plotted in (e), is injected instead, then the output, plotted in (g), would be a scaled and delayed sine wave. Note that the red box in (g) is identical to that in (c). Due to periodicity in $n t$ of the input sine wave, in (g) the black box is identical to the red. 


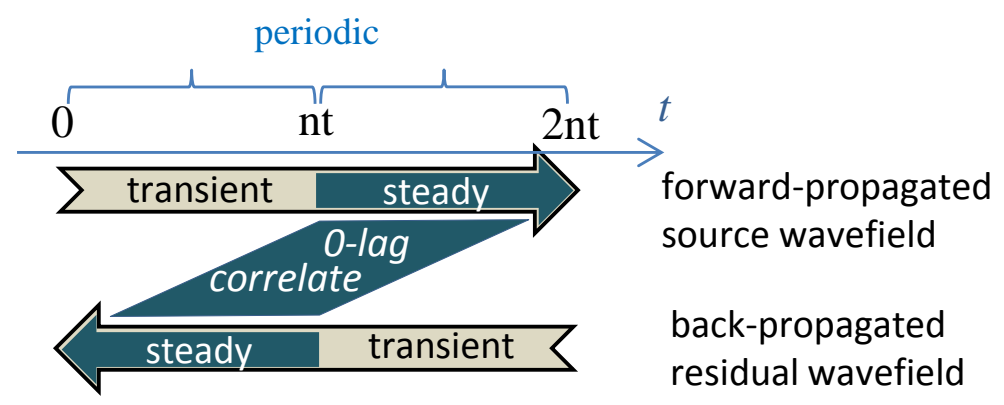

Figure 3: Transient-reduction in forming the gradient of FWI. The residual wavefield is back-propagated over $2 n t$ steps, starting from step $2 n t$. The wavefields for time steps from $n t$ to 1 are steady. The steady wavefields are periodic in time with period $n t$, inherited from the injected sine waves. Therefore, to form the gradient of FWI, we take the 0-lag correlation between the back-propagated residual wavefield (for time slices from 1 to $n t$ ) and forward-propagated source wavefield (for time slices from $n t+1$ to $2 n t$ ). 


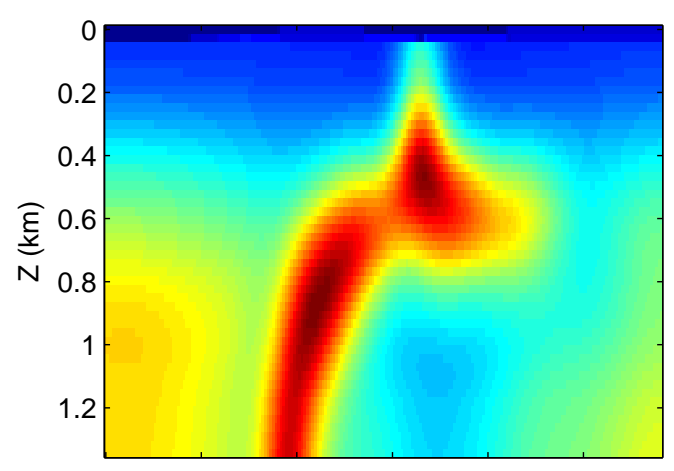

(a) initial velocity

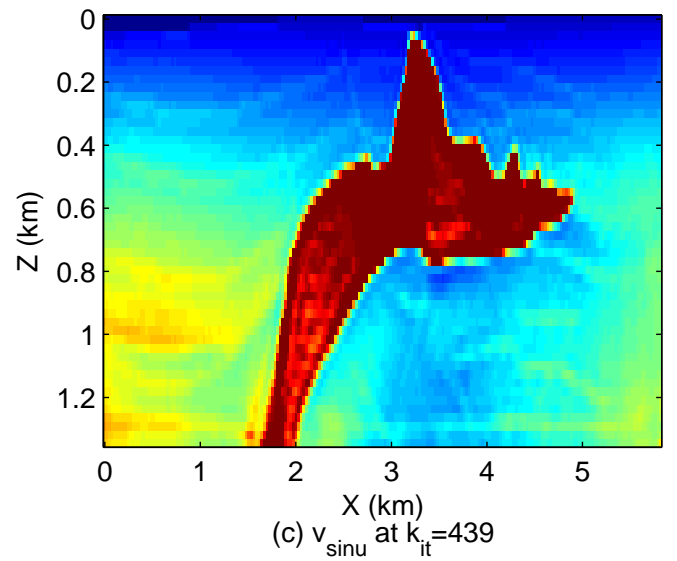

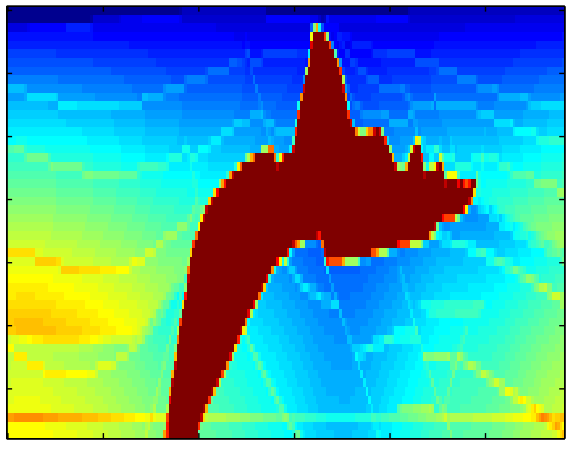

(b) true velocity

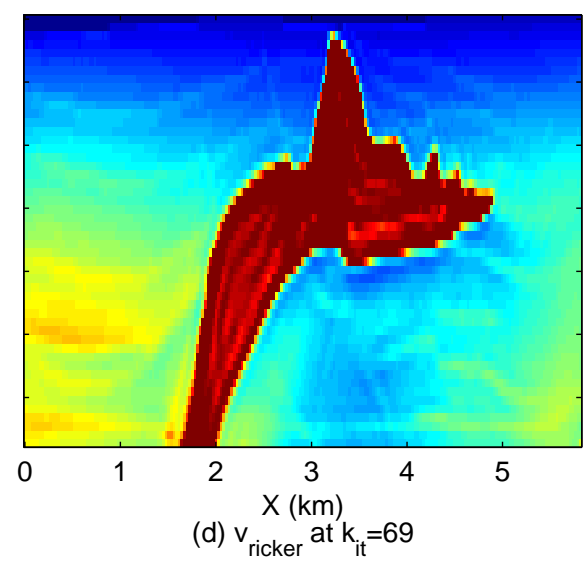

Figure 4: (a) The initial velocity, (b) the true velocity, (c) the result of the proposed FWI at the 439th iteration, and (d) the result of standard FWI at the 69th iteration. 


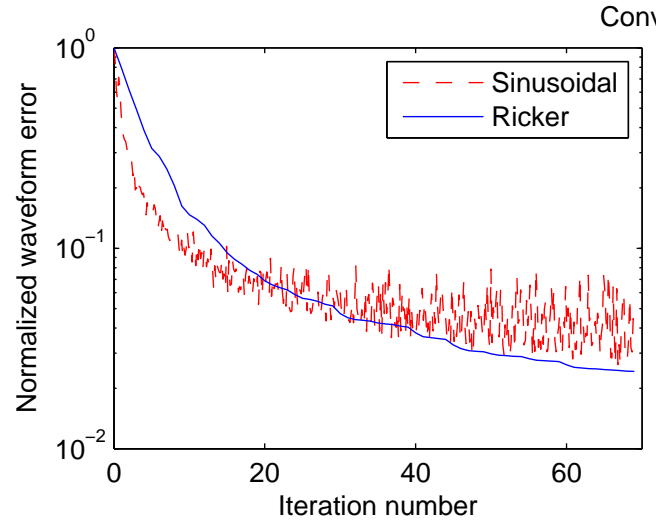

(a)

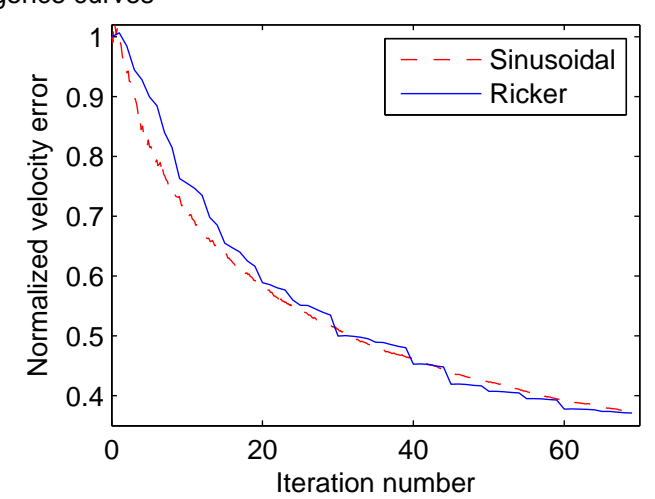

(b)

Figure 5: Normalized error of waveform (a) and velocity (b) as functions of iteration number, for the proposed method (denoted by 'Sinusoidal') and the standard FWI (denoted by 'Ricker'). Note that the iteration numbers for the red dashed curves are actually 6.362 times those in display. See text for details. 


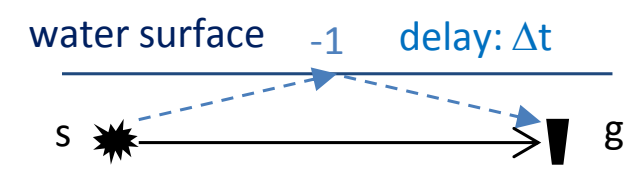

Figure 6: The direct wave and free-surface reflection combine together to give a detected signal approximated by $w(t)-w(t+\Delta t)$, proportional to the time derivative of the wavelet $\mathrm{w}(\mathrm{t})$.

This article is protected by copyright. All rights reserved. 


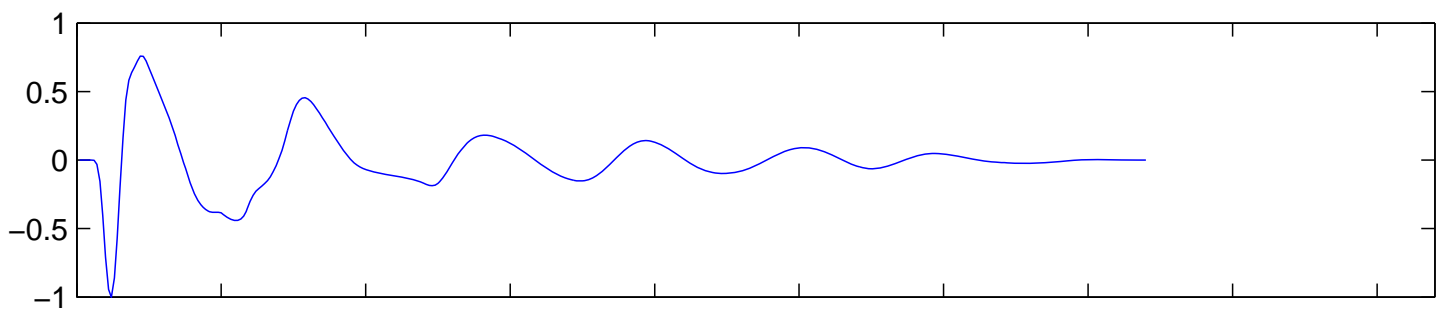

(a)
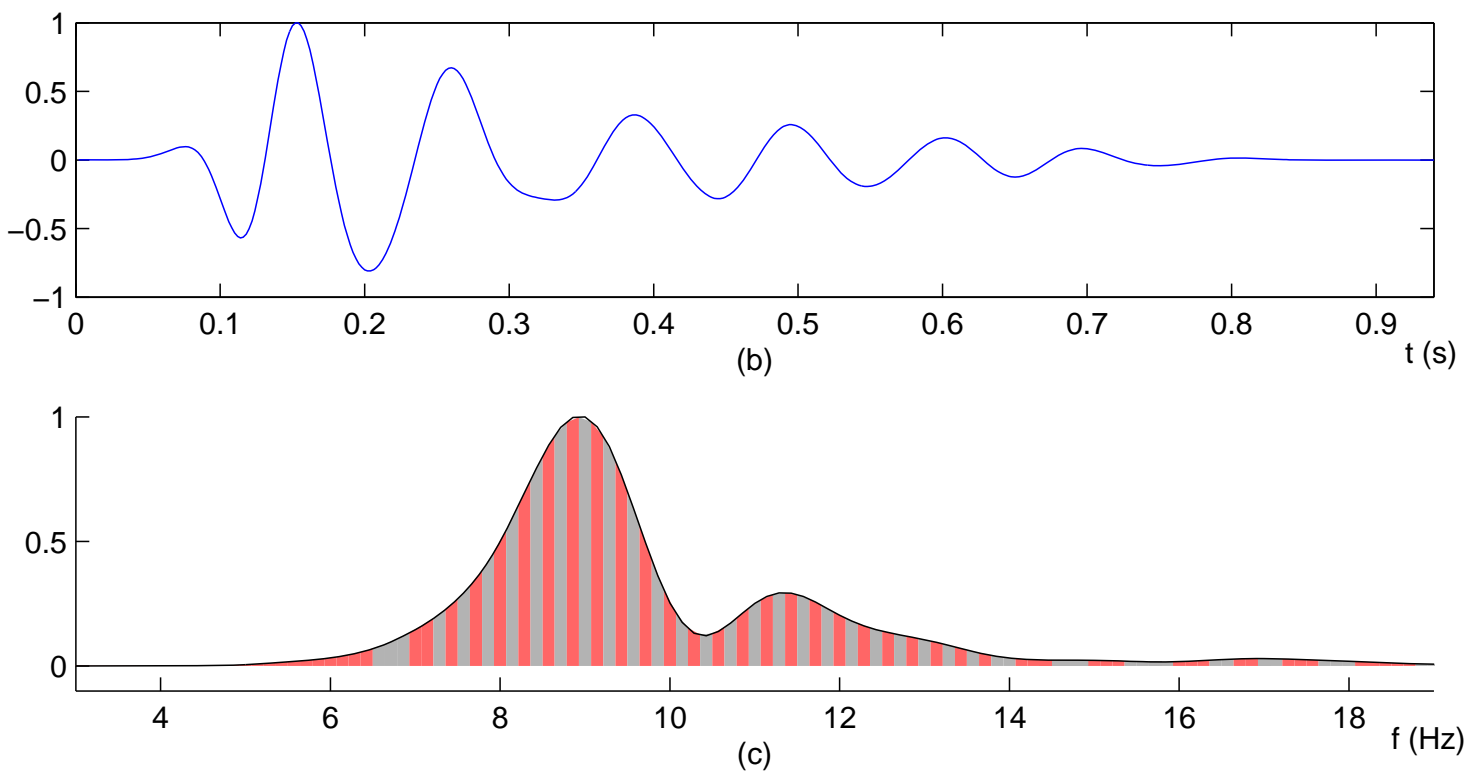

Figure 7: (a) The estimated source wavelet, (b) bandpass filtered wavelet with peak frequency $10 \mathrm{~Hz}$. (c) The power spectrum of (b), which is divided into intervals in order to make the energy of each interval comparable. (Due to discreteness of frequencies, the interval energy cannot be made exactly equal.)

This article is protected by copyright. All rights reserved. 


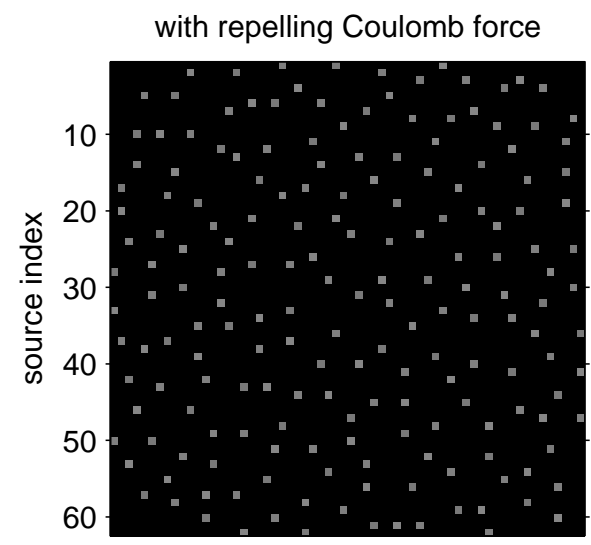

(a)

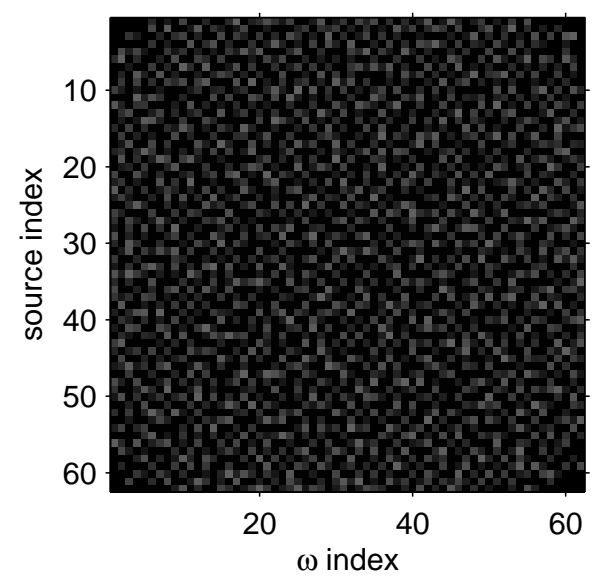

(c)

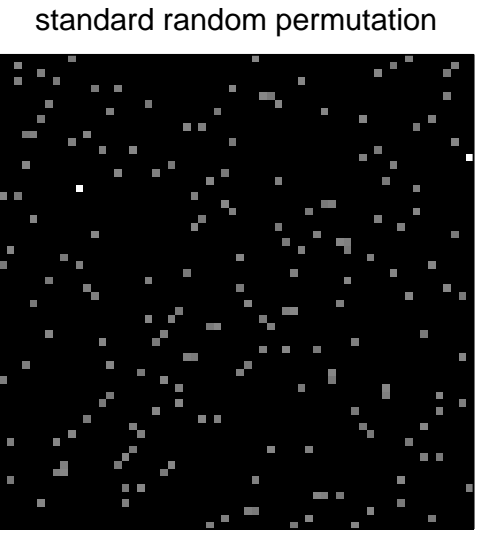

(b)

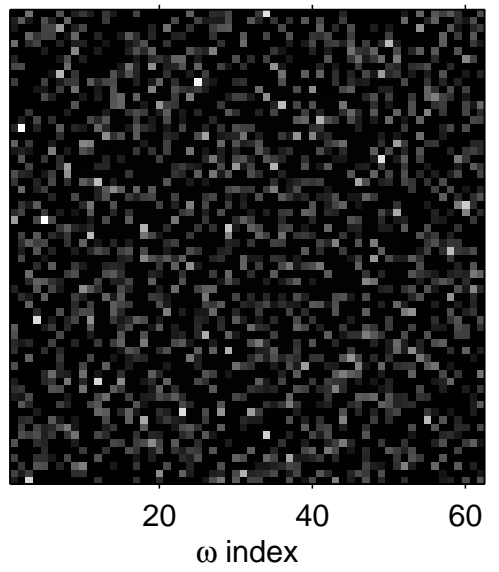

(d)

Figure 8: Frequency-selection encoding results. Each grey spot denotes a mapping between its two coordinates $\omega_{j}$ and $s_{i}$. Upper panels show the mappings accrued over 3 iterations, while lower panels show those over 31 iterations. A bright spot means there are mapping points colliding in history. Earlier mappings are of decayed charges, explaining the varying grey scales of the grey points. 
(a) Traveltime velocity tomogram

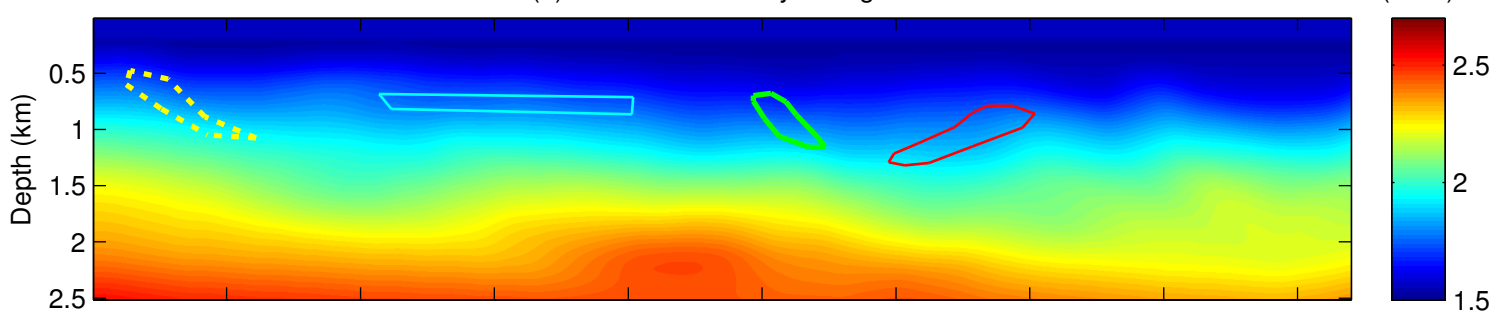

(b) FWI tomogram $(0--25 \mathrm{~Hz})$, using individual sources

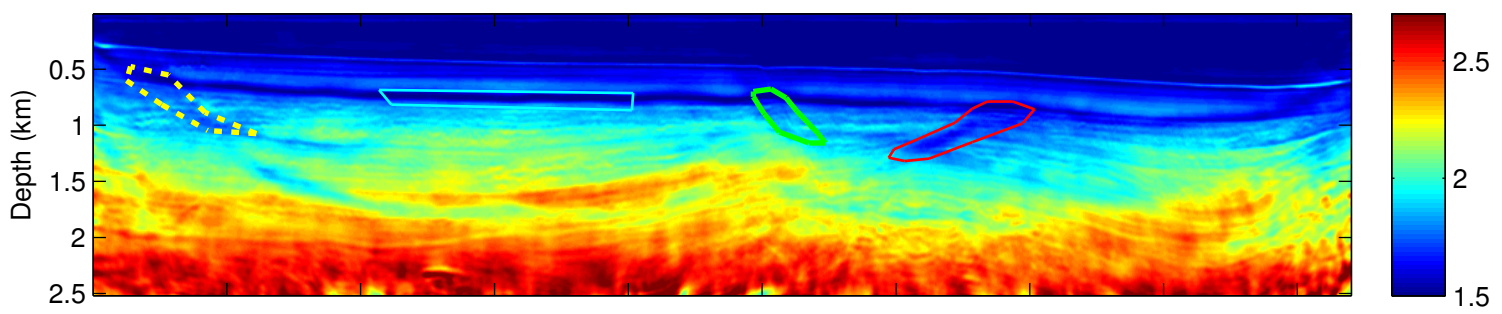

(c) FWI tomogram $(0--25 \mathrm{~Hz})$, using multisource, Quasi-Monte Carlo

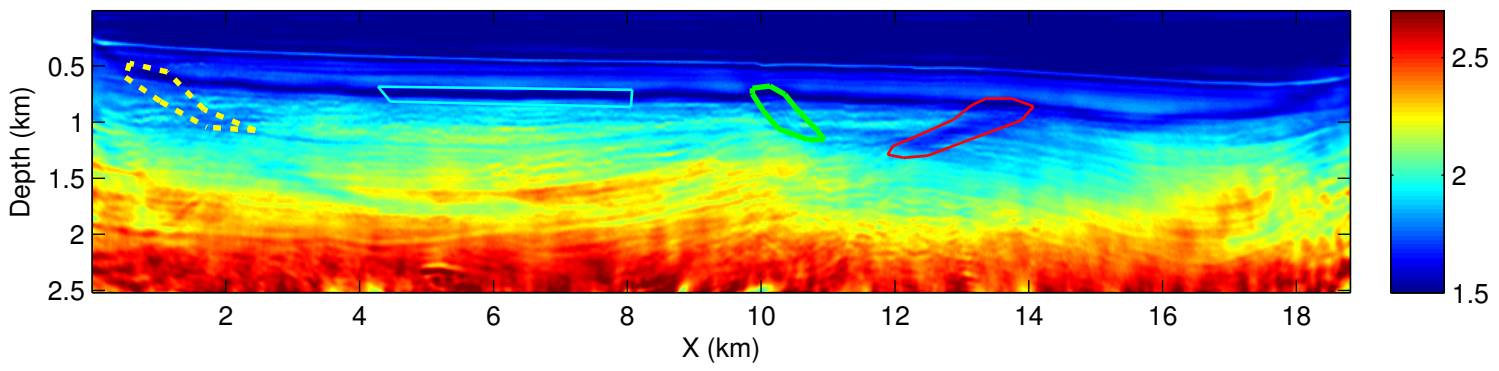

Figure 9: FWI results for the GOM dataset. (a) The initial velocity model. The velocity tomograms obtained from (b) standard FWI after 20 iterations, and (c) FWIMFS after 71 iterations. The encircled regions are low-velocity anomalies in the FWI results. 


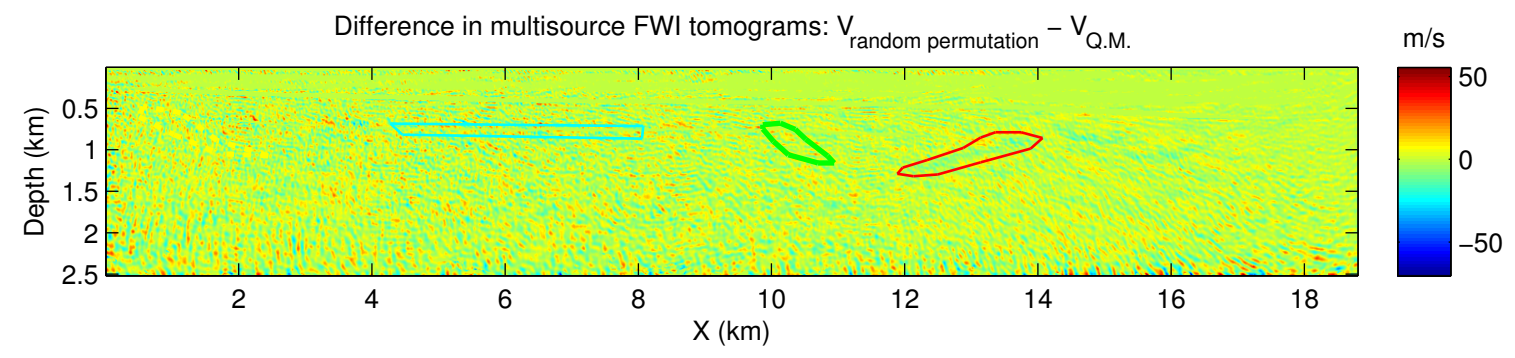

Figure 10: Difference of FWI velocity models obtained using Quasi-Monte Carlo versus standard random permutation in frequency-selection encoding. The encirclements are for reference only.

This article is protected by copyright. All rights reserved. 


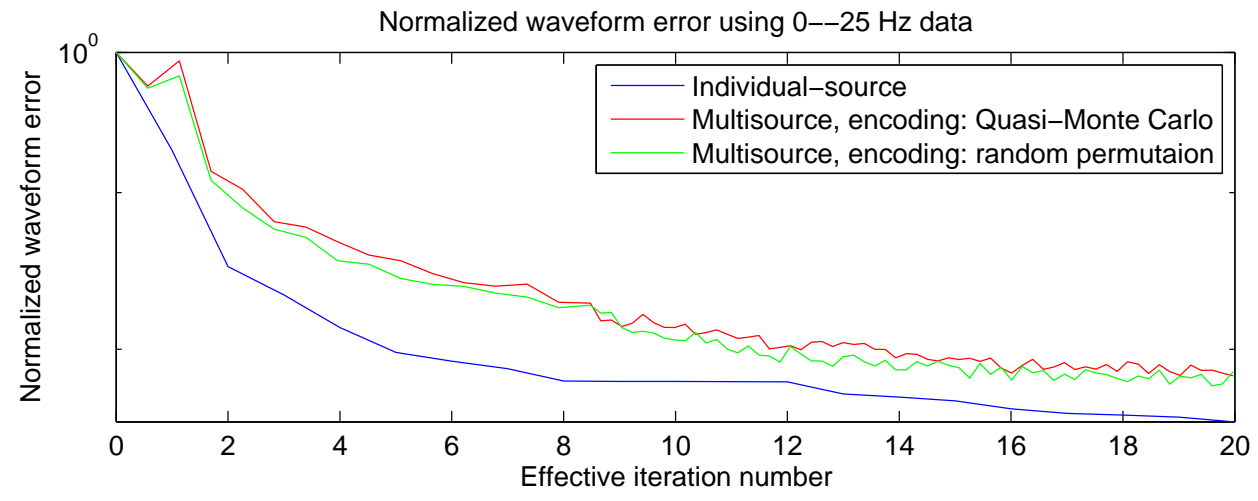

Figure 11: Reduction of data misfit (for 0-25 Hz) plotted against FWI iterations, starting from the model obtained from the $0-15 \mathrm{~Hz}$ data. The iteration numbers are labeled according to those of the standard FWI (i.e., of individual-source). The curves of the multisource cases are scaled horizontally such that when one draws a vertical line across this figure, the intersected red and green points represent $1 / 8$ of the computational cost than the cost of the intersected blue point.

This article is protected by copyright. All rights reserved. 

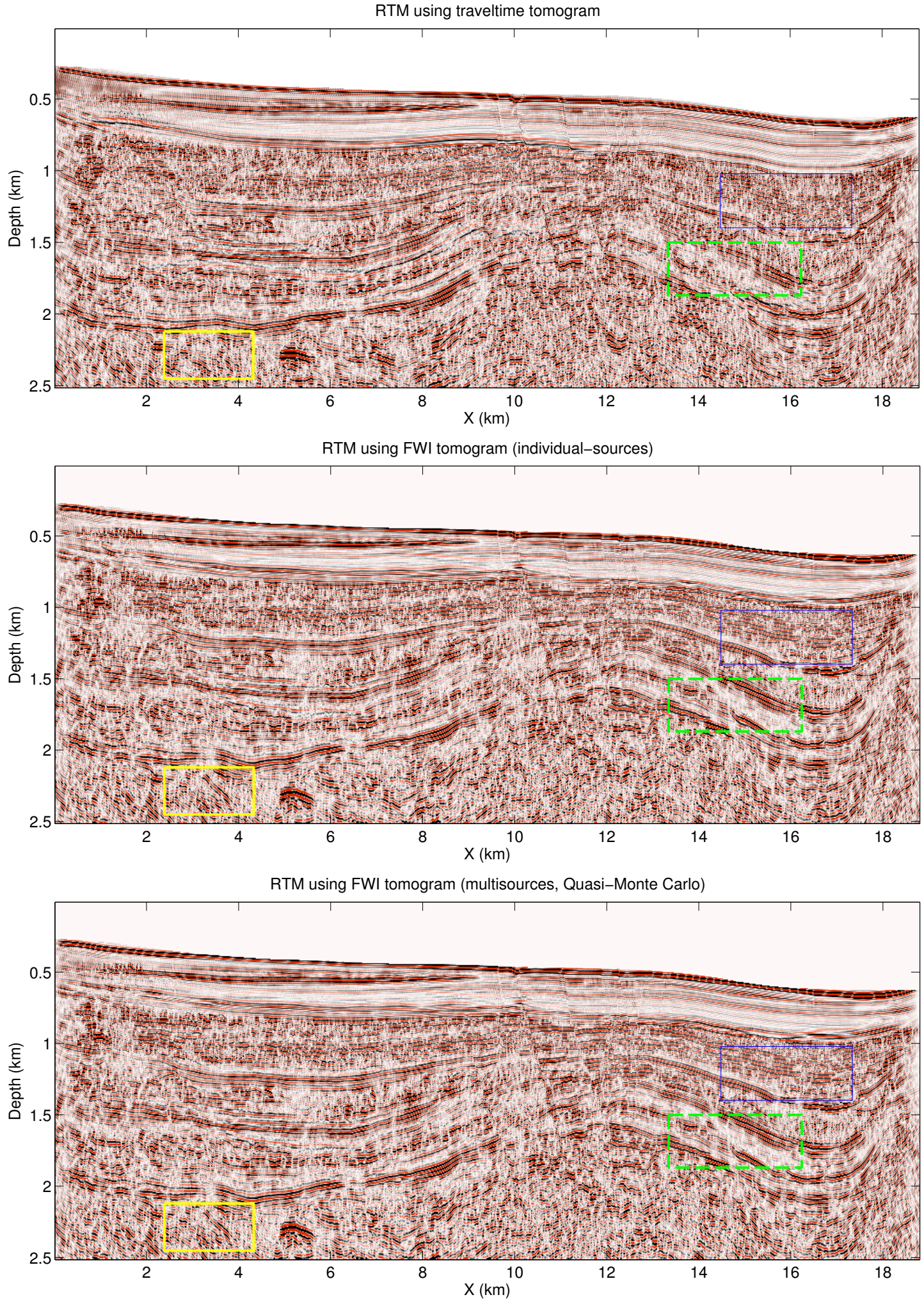

Figure 12: RTM images from the GOM data, when the source wavelet is Wiener-reshaped to a Ricker wavelet of peak frequency $30 \mathrm{~Hz}$. The velocity models (from top panel to bottom) are obtained from traveltime tomography, from standard FWI (after 20 iterations), and from FWIMFS (after 71 iterations). The three colored boxes are for zoomed views in Figure 13.

This article is protected by copyright. All rights reserved. 

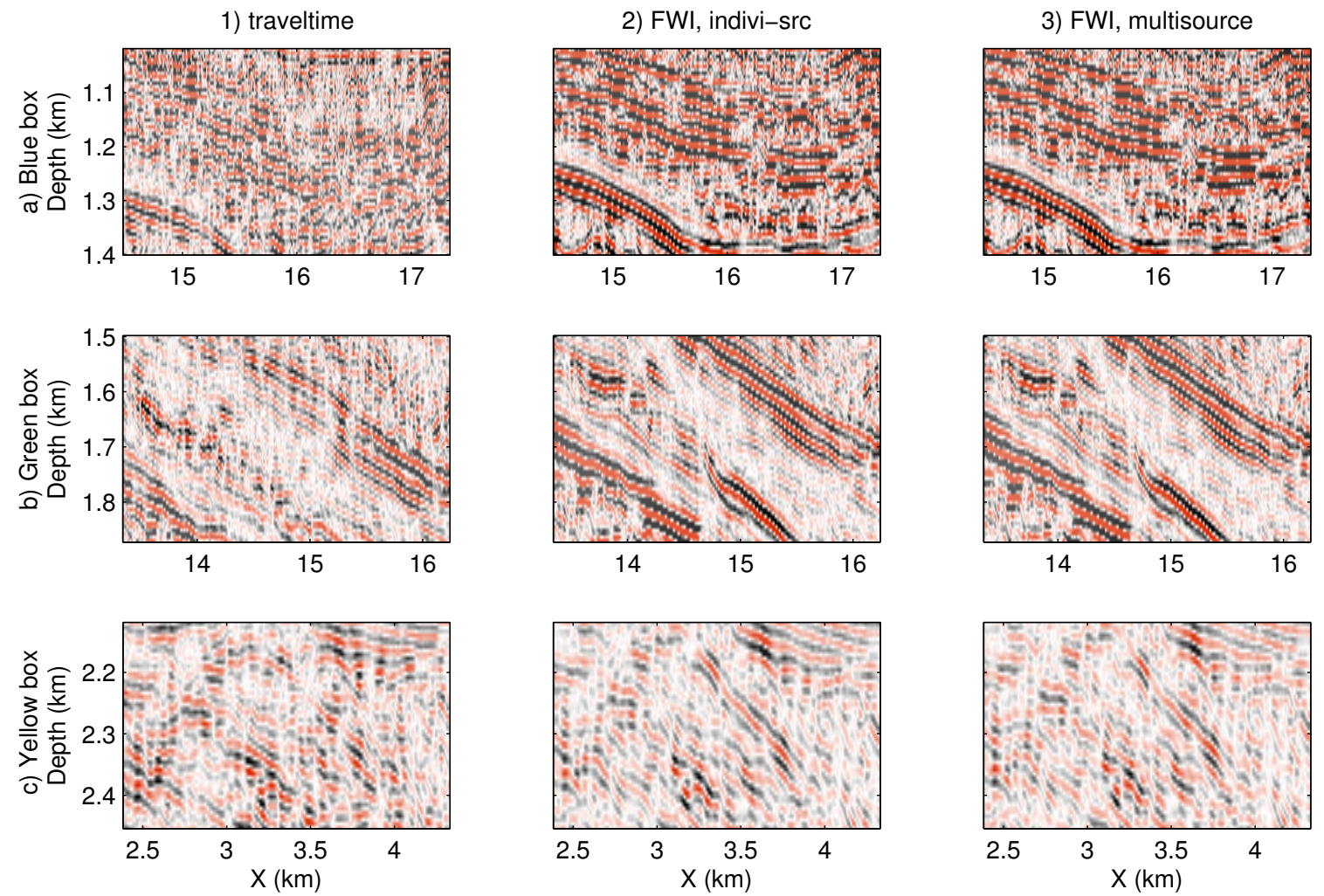

Figure 13: Zoomed views of the RTM images in Figure 12.

This article is protected by copyright. All rights reserved. 
(a) CIGs for traveltime tomogram

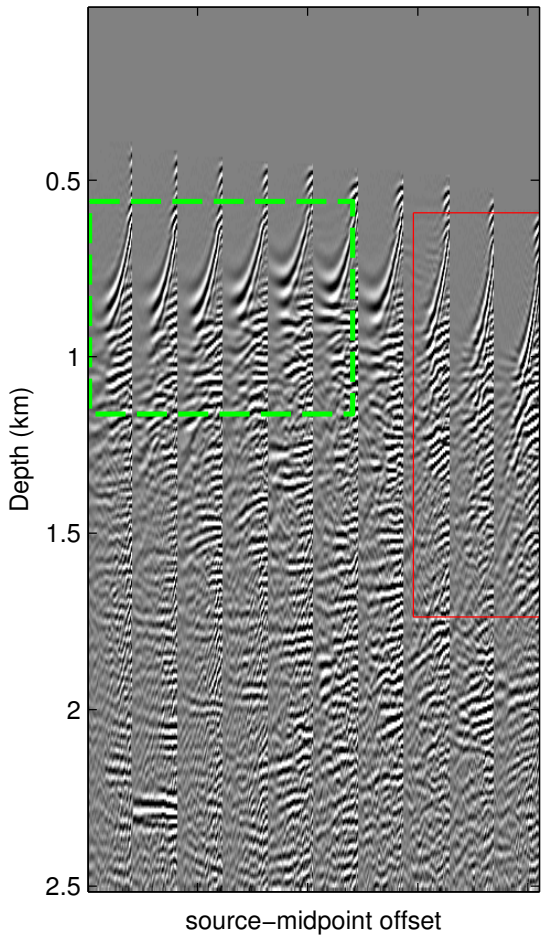

(b) CIGs for FWI, indivi-src

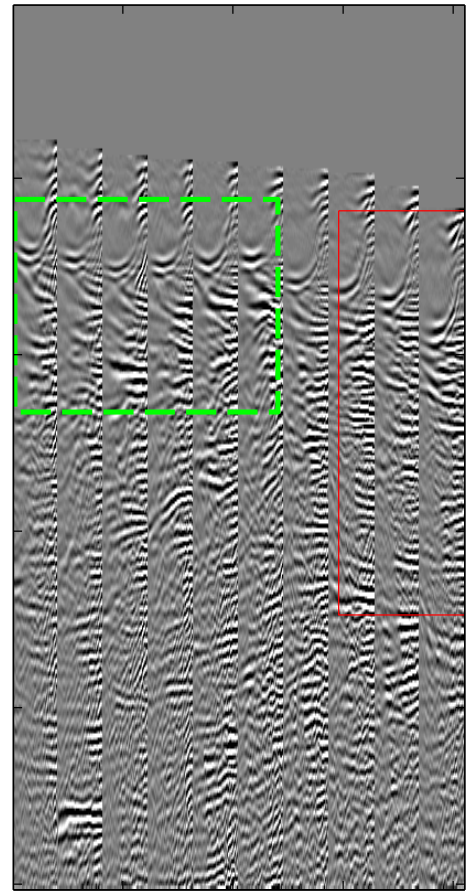

source-midpoint offset (c) CIGs for FWI, multisource

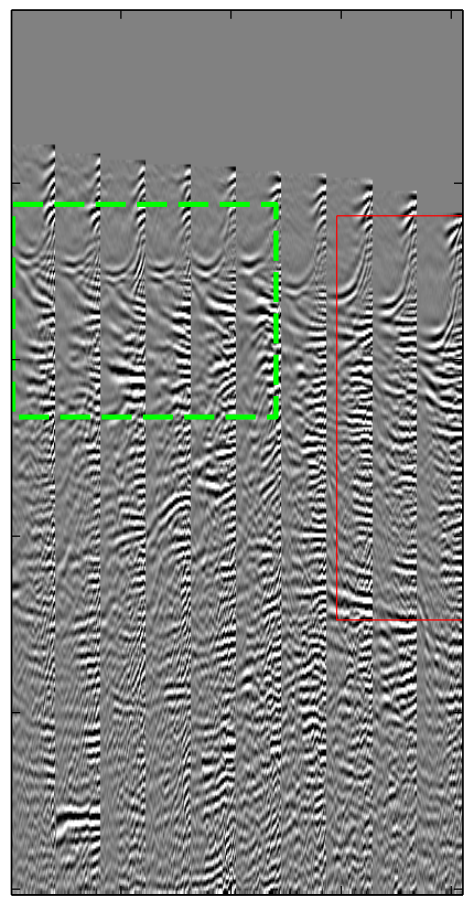

source-midpoint offset

Figure 14: Shot domain CIG. The boxed areas show improved flatness in the FWI results compared to that of the traveltime tomogram.

This article is protected by copyright. All rights reserved. 
(a) observed CSG

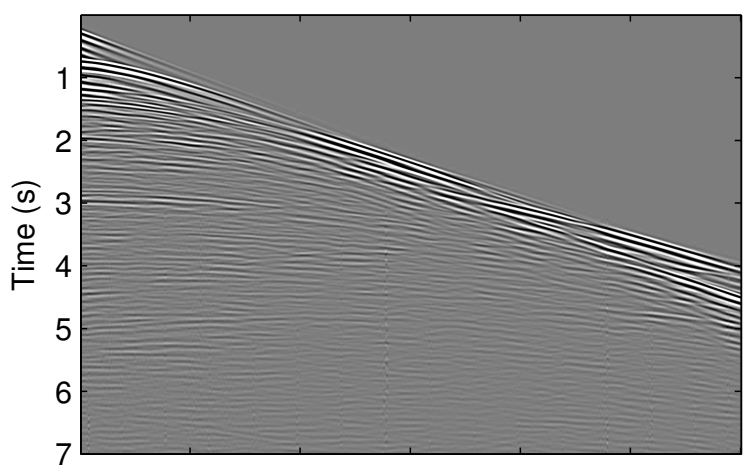

(c) FWI (indivi-src) predicted CSG

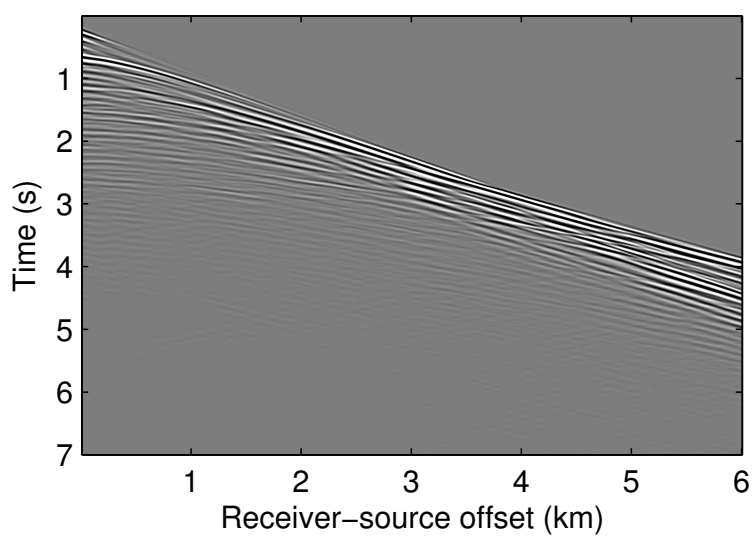

(b) TRT predicted CSG

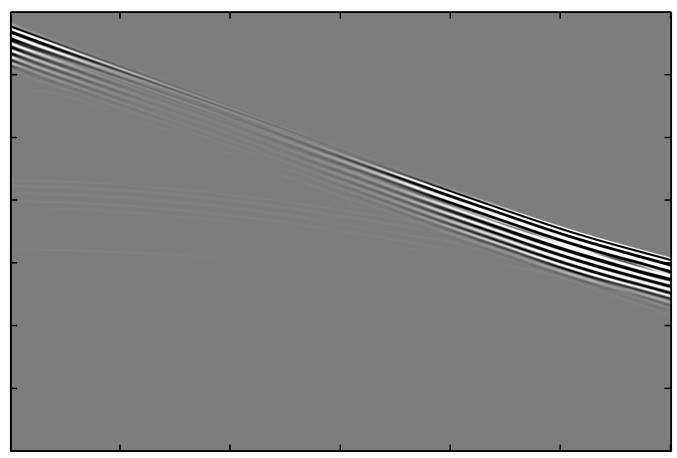

(d) FWI (multisource) predicted CSG

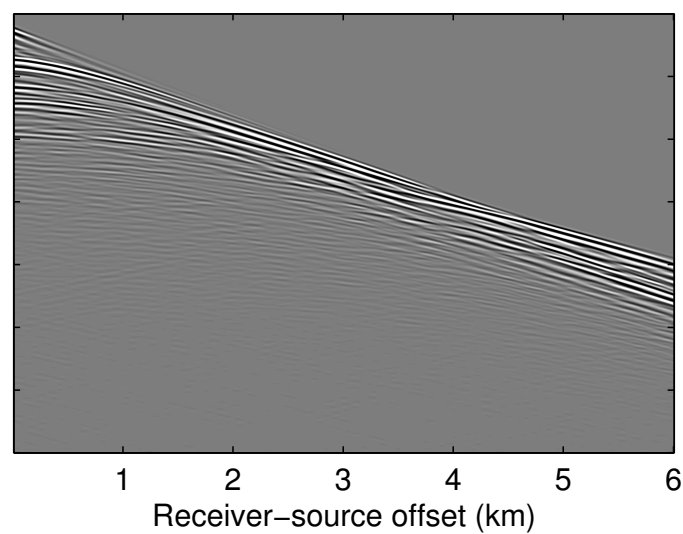

Figure 15: CSG for GOM data of one source at $X=6 \mathrm{~km}$. (a) the observed data bandpass filtered to $0-25 \mathrm{~Hz}$. The predicted counterparts obtained with the (b) traveltime tomograms, (c) standard FWI (after 20 iterations), and (d) FWIMFS (after 71 iterations). 
(a) observed CSG

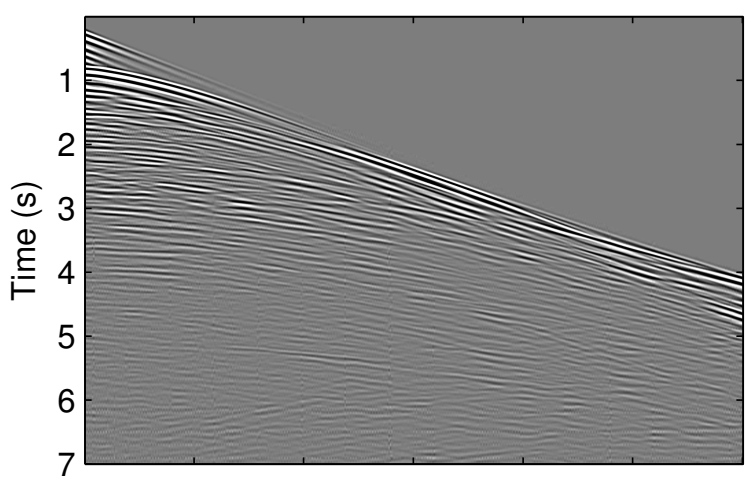

(c) FWI (indivi-src) predicted CSG

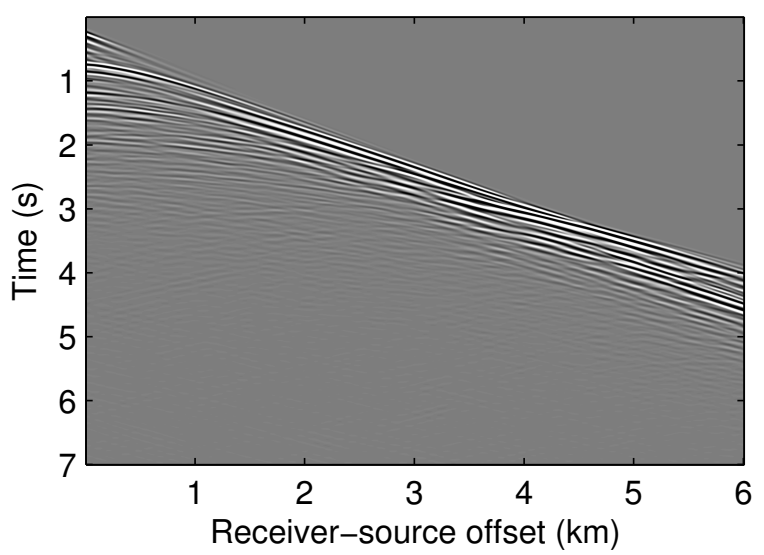

(b) TRT predicted CSG

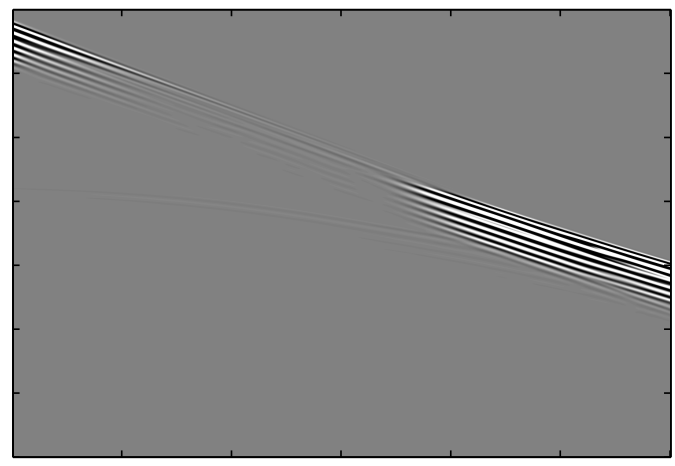

(d) FWI (multisource) predicted CSG

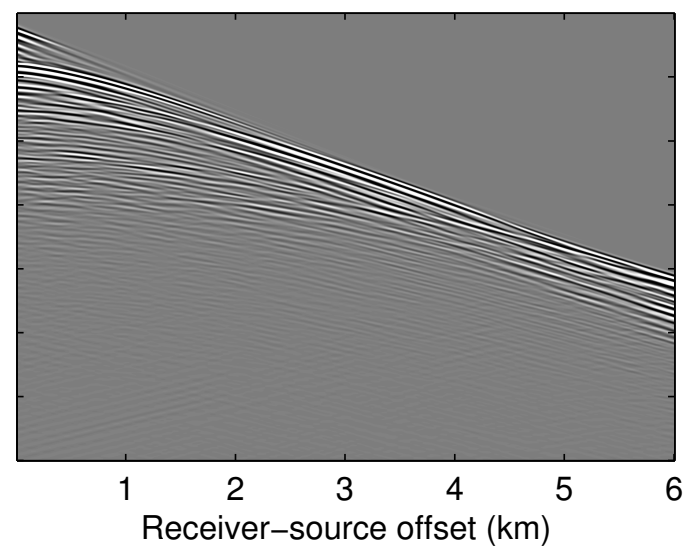

Figure 16: CSG for GOM data of one source at $X=12 \mathrm{~km}$. (a) the observed data bandpass filtered to $0-25 \mathrm{~Hz}$. The predicted counterparts obtained with the (b) traveltime tomograms, (c) standard FWI (after 20 iterations), and (d) FWIMFS (after 71 iterations). 